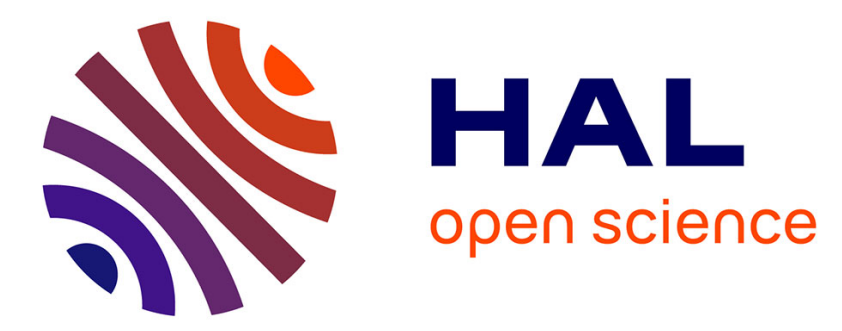

\title{
Closed-form expressions for the total power radiated by an electrically long multiconductor line
}

\author{
Andrea Cozza, Bernard Demoulin
}

\section{To cite this version:}

Andrea Cozza, Bernard Demoulin. Closed-form expressions for the total power radiated by an electrically long multiconductor line. IEEE Transactions on Electromagnetic Compatibility, 2009, 51 (1), pp.119 - 130. hal-00441340

\author{
HAL Id: hal-00441340 \\ https://hal.science/hal-00441340
}

Submitted on 8 Nov 2010

HAL is a multi-disciplinary open access archive for the deposit and dissemination of scientific research documents, whether they are published or not. The documents may come from teaching and research institutions in France or abroad, or from public or private research centers.
L'archive ouverte pluridisciplinaire HAL, est destinée au dépôt et à la diffusion de documents scientifiques de niveau recherche, publiés ou non, émanant des établissements d'enseignement et de recherche français ou étrangers, des laboratoires publics ou privés. 


\title{
Closed-Form Expressions for the Total Power Radiated by an Electrically Long Multiconductor Line
}

\author{
A. Cozza ${ }^{1}$, B. Démoulin ${ }^{2}$ \\ ${ }^{1}$ SUPELEC, Département de Recherche en Électromagnétisme, Gif sur Yvette, France, \\ andrea.cozza@supelec.fr \\ ${ }^{2}$ Université des Sciences et Technologies de Lille, Laboratoire TELICE (IEMN), Lille, France
}

\begin{abstract}
Two analytical solutions based on transmission-line theory for the total power radiated by a multiconductor line above a ground-plane are proposed. The line is not assumed to be electrically short or close to the ground-plane, thus making the proposed model suitable for assessing the emission/immunity of actual transmission-lines employed in industrial contexts such as in the automotive domain, in railway lines and power-distribution lines. The model allows an imperfect ground plane to be considered through the complex-image approximation, together with propagation losses. Numerical and experimental results are provided as a validation, while an empirical rule to assess the accuracy of the results is proposed. The two expressions aim at allowing fast parametric analysis of radiation during the design phase of the electrical and geometrical configuration of an unshielded MTL.
\end{abstract}

\section{INTRODUCTION}

Transmission-lines can act as antennas, thus providing the main gateway for radiated interference/subsceptibility. In particular, in many industrial applications multiconductor transmission-lines (MTLs) are mostly unshielded, so that they play a major role in EMC emission/immunity tests. Recently, several authors have suggested the use of new test procedures to characterize equipments from a radiation view-point. These are based on the measurement of the total radiated power (TRP) [1], [2], a global approach opposed to the classic one of characterizing the maximum field radiated at a certain distance by an equipment under test (EUT). The TRP has been historically measured by several means [3], all of them requiring the complete characterization of the radiation pattern of the EUT which, for electrically large devices, is very sensitive to the geometry and excitation of the EUT. In particular, the presence of field nulls complicates the experimental measurement of the radiation patterns. A simpler and far more effective way of providing an estimation makes use of mode-stirred reverberation chambers (MSRC): a statistical evaluation of the TRP is thus provided and can be related to the maximum radiated field by considerations on the EUT electrical dimensions [4]. Besides, TRP is in itself a fundamental quantity for the characterization of any power-radiating equipment. Currently, it is mostly used in the antenna domain for the characterization of radiation efficiency [5], but the pertinence of this methodology in the EMC domain is bound to grow as the electrical dimensions of EUTs increase.
Although the TRP can be easily measured by means of MSRCs (as shown in Section V), its numerical computation during the design phase requires suitable tools, which could be divided into two groups: 1) analytical results from antenna theory, usually developed for the estimation of the radiation resistance of wire antennas; 2) methods based on a power-balance procedure.

In the first group the radiation resistance, and therefore the TRP, is usually computed by integrating Poynting's vector over a closed surface enclosing the antenna [6]. By approximating the actual current-distribution along the antenna conductors through simple analytical expressions, this double integration can be carried out analytically, thus obtaining a closed-form solution for the TRP. The problem with this approach is that the mathematical expressions involved quickly become unwieldy; a typical way of avoiding this problem is to apply simplifying hypothesis such as assuming the line to be electrically short, or to neglect losses [6], [7]. Unfortunately, such hypothesis are very limiting when these models are applied to problems outside their original context. Currently applied standards require to test radiated emissions beyond $1 \mathrm{GHz}$, where these lines can be no longer approximated as electrically short. To the best of our knowledge, only one attempt has been made to derive a closed-form solution for the TRP of an electrically long line [8]:however, in the approximate solution proposed by Storer et al. the line was assumed to be electrically very close to an ideal ground-plane.

Conversely, power-balance methods estimate the amount of power radiated by a device by assessing the difference between the active power going into its ports and that coming out of them. The amount of power missing from this balance is somehow "lost" inside the device. Considering the case of a transmission-line, this missing power can be associated to two main phenomena: conversion from electric power into thermic one by Joule's effect (ohmic losses) and conversion from electric power into radiating electromagnetic power (radiation losses). By estimating the amount of power lost into ohmic losses, one can easily assess the TRP. Nevertheless, applying it in a modelling problem requires suitable tools. In fact, in order to apply the power balance procedure, a full-wave model of the transmission-line must be used. This task can be achieved by means of two broad approaches: on the one hand numerical models directly solving Maxwell's equations; on the other hand, 


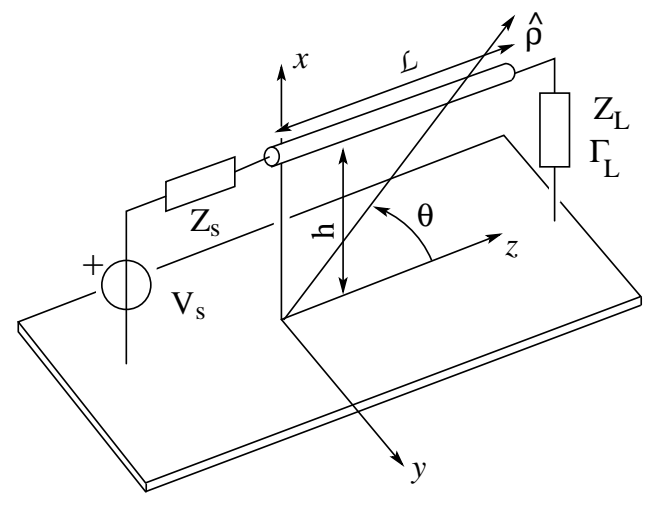

Fig. 1. The transmission-line and its reference system.

by applying analytical models extending the validity of TLT by including radiation losses. This latter approach has been pursued by several authors in recent years [9][12]. Although these techniques provide a very powerful tool for high-frequency modelling of transmission-lines, their actual implementation is far from being accessible to the average industrial user. Conversely, the application of numerical methods may require a significant computationtime in the design phase. Therefore, both these two groups of solutions should be rather applied in a context where high-level skills are available, as well as time is not a constraint.

The aim of this paper is to present an alternative analytical solution for the TRP of an electrically long multiconductor line. In particular, the basic idea is to provide an estimation of the TRP from a TLT description of an MTL: this approach is interesting since TLT does not require high-level skills, so that it would lead to a simpler implementation. Furthermore, engineers dealing with transmission line problems already make use of TLT modelling, so that the proposed model would appear as a sort of natural extension for the assessment of the TRP. Since the proposed model deals with open lines, it can be directly applied not just to the automotive domain, but also for the assessment of the radiation efficiency of interferences propagating along railway lines, or that of high-frequency telecommunication signals in powerline communications. Such an analytical model, although inevitably affected by limitations, could be effectively employed in parametric analyses of the TRP for a line, much faster than with a numerical model. This would allow to identify the most interesting configurations (e.g. worst-cases or best ones) and to apply full-wave models just to a smaller subset of configurations.

\section{THE MODEL}

The system considered here is a uniform multiconductor line of finite length $\mathscr{L}$, located above an infinite ground-plane. For the sake of simplicity, the case of a single-wire line will be considered first (Fig. 1). The proposed analytical solution is based upon the following hypotheses:

1) the thin-wire approximation holds for the overhead conductor,
2) the ground-plane is boundless,

3) the medium in which the line is immersed is lossless,

4) the current-distribution along the line can be described as the sum of two complex exponentials, and

5) the radiation due to the discontinuities introduced by the line ends (e.g. vertical risers) is assumed to be negligible, i.e., the line is regarded as the main source of radiation or as the main gateway for coupling to radiated interferences.

Hypothesis 4) implies that the propagation along the line can be described through transmission-line theory. This poses a limitation to the highest frequency that can be covered by the model. This also implies that radiation losses should be negligible, i.e., radiation modes should not be strongly excited with respect to transmission-line ones or, in other words, that the line is not acting as an efficient antenna [13]. Since transmission-line modes are deemed to be dominant, the effect of radiation losses (due to the excitation of radiation modes) can be accounted for through a perturbation of the transmission-line solution. This is the same approach used when including losses into waveguide structures, by keeping the same modal solution as for the lossless case, while introducing a modification of the propagator function accounting for the losses. This perturbation approach allows extending the validity of the transmission-line model to higher frequencies, as we will show for the single-wire case, by means of a two-step procedure (see Section V), by giving an estimation of the effect of radiation losses in the computation of the TRP.

Following point 5), the loads and the voltage generator in Fig. 1 should be just regarded as mathematical boundary conditions rather than physical connections. The closed-form solutions here proposed cannot take into account the contribution coming from external components, such as vertical risers.

The TRP is usually introduced in antenna theory for the definition of the radiation resistance of wire-antennas [6] and defined as

$$
P_{r}=\frac{1}{2} \operatorname{Re} \iint_{\Omega} \mathbf{S} \cdot \hat{\boldsymbol{n}} \mathrm{d} \Omega,
$$

where $\Omega$ is a closed bounding-surface embracing the entire line, $\mathbf{S}$ is Poynting's vector and $\hat{\boldsymbol{n}}$ is the unitary outward vector normal to the surface $\Omega$. In the case of an ideal ground-plane, this surface can be reduced to an hemisphere over the ground-plane, the electromagnetic (EM) field being identically equal to zero beneath the metallic boundary. For a lossy ground-plane, this hypothesis is not fully met, since part of the EM energy radiated by the line is directed into the ground-plane. In the context of this work, the fraction of energy radiated into the ground-plane will be assumed to be negligible, as compared to the energy radiated into the upper-space. This hypothesis is just applied to the EM field that is not guided by the line: part of the transversal topography of the propagating modes is actually inside the groundplane. Hence, the losses due to propagation over a lossy ground-plane can be included into the model through the per-unit-length (p.u.1.) parameters of the line [14]. 
Assuming the upper medium to be lossless, (1) can be recast in the far-field as follows:

$$
P_{r}=\frac{1}{2} \iint_{\Omega} \zeta_{0}|\mathbf{H}(\rho, \vartheta, \varphi)|^{2} \mathrm{~d} \Omega,
$$

where $\zeta_{0}$ is the characteristic impedance of the surrounding medium. Variables $\rho, \vartheta$ and $\varphi$ refer to Fig. 1: $\vartheta$ is the angle between the direction $\hat{\rho}$ and the axis $z$, whereas $\varphi$ is the azimuthal angle considered on the $x y$ plane. Due to hypothesis 4 ), the following modal description for the current-distribution has been used:

$$
I(z)=I_{0}^{+}\left(\mathrm{e}^{-\gamma z}-\Gamma_{\mathrm{L}} \mathrm{e}^{-2 \gamma \mathscr{L}} \mathrm{e}^{+\gamma z}\right),
$$

where $I_{0}^{+}$is the forward-travelling modal current, $\gamma=$ $\gamma_{0} \xi$ is the propagation constant for the wave guided by the line, and $\Gamma_{L}$ represents the load reflection coefficient. The parameter $\gamma_{0}=\mathrm{j} k_{0}$ is the propagation constant of the surrounding medium, so that $\xi=\xi^{\prime}-\mathrm{j} \xi^{\prime \prime}$ acts as a sort of equivalent permittivity for the propagation along the line.

The computation of the radiated field can be greatly simplified by invoking the image principle, thus removing the lower half-space. Although it is usually employed just for perfect electric conductors, this concept has been extended to lossy conductors through the use of the complex-image method [15], by placing the image at a complex distance $h_{C I}=h+\mathrm{j} \delta$ below the groundplane, where $\delta$ is the skin-effect penetration depth for the ground-plane material. Thus, the original line can be analyzed as a two-wire line in a homogeneous medium, with wires running at a distance $h_{C I}+h=2 h^{\prime}$, where $h^{\prime}=h+\mathrm{j} \delta / 2$.

A classical approach from antenna theory [16] can then be applied, by integrating over the current-distribution $I(z)$, plus its image, to compute the far-field expression of the magnetic field:

$$
\begin{aligned}
\mathbf{H}(\rho, \vartheta, \varphi)= & \hat{\boldsymbol{\varphi}} \frac{k_{0}}{2 \pi \rho} \sin \vartheta \sinh \left(k_{0} h^{\prime} \hat{\boldsymbol{x}} \cdot \hat{\boldsymbol{\rho}}\right) \mathrm{e}^{-\gamma_{0} \rho} . \\
& \cdot \int_{0}^{\mathscr{L}} I(z) \mathrm{e}^{-\gamma_{0} z \hat{\boldsymbol{z}} \cdot \hat{\boldsymbol{\rho}}} \mathrm{d} z
\end{aligned}
$$

The projection terms $\hat{\boldsymbol{x}} \cdot \hat{\boldsymbol{\rho}}$ and $\hat{\boldsymbol{z}} \cdot \hat{\boldsymbol{\rho}}$ take into account the phase-shift of the contribution of each elementary source along the radiation direction $\hat{\rho}$. Inserting this result into (2) and expressing $\mathrm{d} \Omega$ in spherical coordinates yields:

$$
\begin{aligned}
P_{r}= & \frac{\left|I_{0}^{+}\right|^{2} \zeta_{0}}{4 \pi^{2}} \int_{0}^{\pi} \int_{-\pi / 2}^{+\pi / 2} \frac{\sin ^{3} \vartheta}{\left|\xi^{2}-\cos ^{2} \vartheta\right|^{2}} . \\
& \cdot\left|\sinh \left(k_{0} h^{\prime} \sin \vartheta \cos \varphi\right)\right|^{2}|B(\vartheta)|^{2} \mathrm{~d} \varphi \mathrm{d} \vartheta
\end{aligned}
$$

with

$$
\begin{aligned}
B(\vartheta) & =\left(\mathrm{e}^{-\gamma_{0}(\cos \vartheta+\xi) \mathscr{L}}-1\right)(\cos \vartheta-\xi)+ \\
& -\Gamma_{\mathrm{L}} \mathrm{e}^{-2 \gamma \mathscr{L}}\left(\mathrm{e}^{-\gamma_{0}(\cos \vartheta-\xi) \mathscr{L}}-1\right)(\cos \vartheta+\xi) .
\end{aligned}
$$

After some algebraic manipulations, (6) yields:

$$
\begin{aligned}
|B(\vartheta)|^{2} & =A_{1}+ \\
& +A_{2} \sin ^{2} \vartheta+ \\
& +A_{3} \cos \left(k_{0} \mathscr{L} \cos \vartheta\right)+ \\
& +A_{4} \sin ^{2} \vartheta \cos \left(k_{0} \mathscr{L} \cos \vartheta\right)+ \\
& -A_{5} \cos \vartheta \sin \left(k_{0} \mathscr{L} \cos \vartheta\right),
\end{aligned}
$$

where the five coefficients $A_{i}$ are given below:

$$
\begin{aligned}
& A_{1}=\left(1+|\xi|^{2}\right)\left(1+\chi^{2}\right) \sigma-4 \chi^{2} \tau \kappa_{c}\left(1-|\xi|^{2}\right) \\
& A_{2}=4 \chi^{2} \tau \kappa_{c}-\sigma\left(1+\chi^{2}\right) \\
& A_{3}=2 \chi\left[2 \tau \chi P_{c}\left(1-|\xi|^{2}\right)-\kappa_{c} \sigma\left(1+|\xi|^{2}\right)\right] \\
& A_{4}=2 \chi\left(\kappa_{c} \sigma-2 \chi \tau P_{c}\right) \\
& A_{5}=4 \chi\left(\xi^{\prime} \sigma \kappa_{s}+2 \chi \xi^{\prime \prime} P_{s} \tau\right)
\end{aligned}
$$

with

$$
\begin{aligned}
\tau & =\operatorname{Re}\left\{\Gamma_{\mathrm{L}} \mathrm{e}^{-\mathrm{j} k_{0} \xi^{\prime} \mathscr{L}}\right\} \\
\chi & =\mathrm{e}^{-k_{0} \xi^{\prime \prime} \mathscr{L}} \\
\sigma & =1+\left|\Gamma_{\mathrm{L}}\right|^{2} \\
\kappa_{c} & =\cos \left(k_{0} \xi^{\prime} \mathscr{L}\right) \\
\kappa_{s} & =\sin \left(k_{0} \xi^{\prime} \mathscr{L}\right) \\
P_{c} & =\cosh \left(k_{0} \xi^{\prime \prime} \mathscr{L}\right) \\
P_{s} & =\sinh \left(k_{0} \xi^{\prime \prime} \mathscr{L}\right) .
\end{aligned}
$$

In order to solve (5), the double-integral has to be factorized into two one-dimensional integrals. An easy and straightforward solution is to employ the McLaurin series expansion of $\sinh ^{2} x$, which eventually yields

$$
P_{r}=\frac{\left|I_{0}^{+}\right|^{2} \zeta_{0}}{4 \pi^{2}} \sum_{n=1}^{\infty} a_{n} \int_{0}^{\pi} \frac{\sin ^{2 n+3} \vartheta}{\left|\xi^{2}-\cos ^{2} \vartheta\right|^{2}}|B(\vartheta)|^{2} \mathrm{~d} \vartheta
$$

with

$$
\begin{aligned}
& a_{n}=b_{n}+c_{n} \\
& b_{n}=-\left(\frac{k_{0} h}{n}\right)^{2} b_{n-1} \\
& c_{n}=\left(\frac{k_{0} \delta}{2 n}\right)^{2} c_{n-1},
\end{aligned}
$$

where $b_{0}=-\pi / 2$ and $c_{0}=\pi / 2$. The terms in the integral in (20) share the same structure which, though being analytically solvable, has no simple closed-form solution. Nevertheless, the special cases $\xi=1$ and $\xi^{2}-\cos ^{2} \vartheta \simeq \xi^{2}$ provide a closed-form result [17], which is exact for $\xi=1$ :

$$
\begin{aligned}
P_{r} & \simeq \frac{\left|I_{0}^{+}\right|^{2} \zeta_{0}}{8 A_{0} \pi^{2}} \sum_{n=K+1}^{\infty} a_{n-K}\left\{A_{1} S_{2 n-1}+\right. \\
& +A_{2} S_{2 n+1}+ \\
& +A_{3} \psi_{n}+ \\
& \left.+\left(A_{4}-\frac{k_{0} \mathscr{L}}{2 n} A_{5}\right) \psi_{n+1}\right\}
\end{aligned}
$$

with

$$
\begin{aligned}
S_{n} & =\int_{0}^{\pi} \sin ^{n} \vartheta \mathrm{d} \vartheta=\frac{n-1}{n} S_{n-2}, \text { and } \\
\psi_{n} & =\frac{\mathrm{J}_{n-1 / 2}\left(k_{0} \mathscr{L}\right)}{\left(k_{0} \mathscr{L} / 2\right)^{n-1 / 2}} \sqrt{\pi}(n-1) !,
\end{aligned}
$$

where $K=0, A_{0}=1$ for the approximation $\xi \simeq 1$ and $K=2, A_{0}=|\xi|^{4}$ otherwise. This result can be simplified by neglecting the Bessel terms $\psi_{n}$, in the case of an electrically long line $\left(k_{0} \mathscr{L}>1\right)$, as shown in Fig. 2a. This implies that (24) is dominated by the load $\Gamma_{\mathrm{L}}$ and 

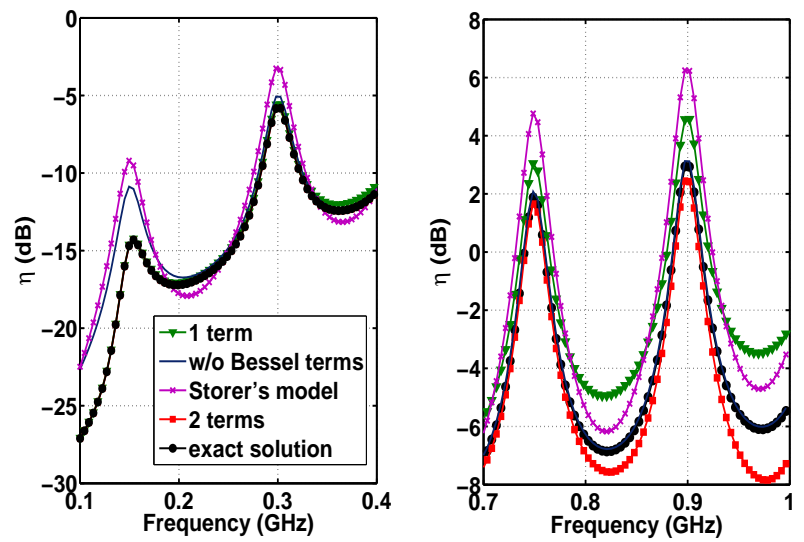

(a)

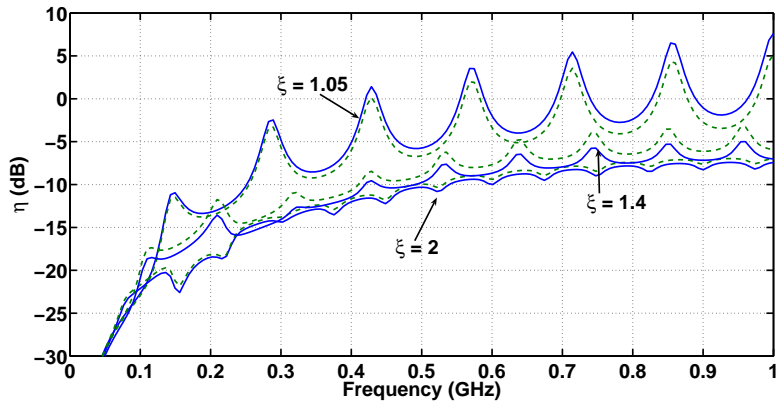

(b)

Fig. 2. Convergence and accuracy tests for expression (24). Picture (a) shows the TRP for a line with $\mathscr{L}=1 \mathrm{~m}, h=10 \mathrm{~cm}, Z_{L}=50 \Omega$ and $\xi=1$ as computed by numerical integration of definition (2) and through the analytical solution (24) for one and two expansion terms. Picture (b) shows the accuracy of (24) when used for $\xi \neq 1$. Dashed lines represent the TRP as computed through numerical integration of (2), solid ones are for expression (24).

especially by the line height $h$, whereas the line length acts just on the position of TRP peaks through $\tau$ and on propagation losses through $\chi$.

In order to check the validity of (24) as a solution for (2), it was compared with the numerical integration of the latter, performed by using an algorithm based on Lobatto's adaptive quadrature. In particular, this check is important for verifying whether the series expansion is fast-convergent or not, as well as for assessing the solution performance when approximating a line with $\xi>1$. To this end, a single-wire line with $h=10 \mathrm{~cm}, \mathscr{L}=1 \mathrm{~m}$, $Z_{s}=Z_{L}=50 \Omega$, with $V_{s}$ providing an available power $P_{\mathrm{av}}=1 \mathrm{~W}$ was considered, over a frequency range up to $1 \mathrm{GHz}$. This means that around $1 \mathrm{GHz}$ the line would be some three wavelengths long, and with $h / \lambda \simeq 1 / 3$.

The first check, involving the series convergence, considered the case $\xi=1$. The TRP will be hereafter normalized to the available power, yielding the radiation efficiency $\eta=P_{r} / P_{\mathrm{av}}$ of the line. The results are shown in Fig. 2a, where the TRP obtained through numerical integration is compared with approximate solutions considering just one term and two terms with a further simplification discarding the terms related to Bessel's functions. Figure 2a demonstrates the good convergence properties of the proposed solution, together with the fact that even the first term provides a good estimate of the TRP. Figure 2 a also shows how (24) gives better results than the model proposed by Storer et al. [8]. Indeed, for an electrically short line [8] neglects the contribution of the Bessel terms, whereas for an electrically long line the first term in (24) gives a better approximation of the TRP, in particular around the peak values. The two-term solution is indistinguishable from the exact solution in the lower frequency-range.

The second check was related to the approximate solution for $\xi>1$. In this case (24) provides very good results even for $\xi=1.4$ (Fig. 2b), which does not fully satisfy the condition $\xi^{2}-\cos ^{2} \vartheta \simeq \xi^{2}$. On the other hand, whenever $\xi \simeq 1$, it is possible to use (24) under the solution for $\xi=1$. An example is shown in Fig. 2 b, where the actual case $\xi=1.05$ was approximated by using the solution for $\xi=1$ : the worst-case error is smaller than $2 \mathrm{~dB}$. The ability of the solution to extend its agreement with the numerical solution for $\xi>1$, will be shown to be very useful for applications to actual lines (see Section VI).

\section{INCLUSION OF RADIATION LOSSES: THE TWO-STEP PROCEDURE}

The very idea of a non-negligible TRP implies that part of the power propagating along the line is radiated away. It was proven in [18] that the propagation along an open line over an ideal ground-plane can be fairly described by a single TEM mode as long as the ratio $h / \lambda \lesssim 1 / 3$.

The problem with this approach is that the attenuation due to radiation losses has to be assessed. Being related to the radiated field, the radiation losses are dependent on the entire current-distribution. This means that it does not suffice just to know the line cross-section as for p.u.l. parameters: therefore, they cannot be generally treated as ohmic losses.

Anyway, for the special case of an infinitely long uniform line, the radiation losses act as ohmic losses [10], so that a p.u.l. radiation resistance $r_{r}=R_{r} / \mathscr{L}$ can be defined and included into the p.u.l. impedance, thus accounting for radiation losses. The same procedure can be pursued for a finite-length line [19], as long as all types of fringing effects can be neglected: in this case the line finiteness is taken into account by the current-distribution itself, which includes a backward-travelling wave. Hence, we just need to compute the radiation resistance $R_{r}$ : this is done in antenna theory by relating the current distribution to the TRP. The following definition [8] will be considered:

$$
R_{r}=\frac{4}{\left(1+\left|\Gamma_{\mathrm{L}}\right|\right)^{2}} \frac{P_{r}}{\left|I_{0}^{+}\right|^{2}}
$$

referring to the current-distribution (3) used as an initial guess, i.e., assuming no radiation losses. In fact, even though this current-distribution does not necessarily suit the actual current-distribution, it provides a fairly good estimate of $R_{r}$. Now it is possible to compute the new propagation constant as:

$$
\gamma=\sqrt{\left(z_{e}+r_{r}\right) y_{e}}
$$

where $z_{e}$ and $y_{e}$ are, respectively, the external p.u.l. impedance and admittance of the line, defined under a quasi-TEM approximation. 


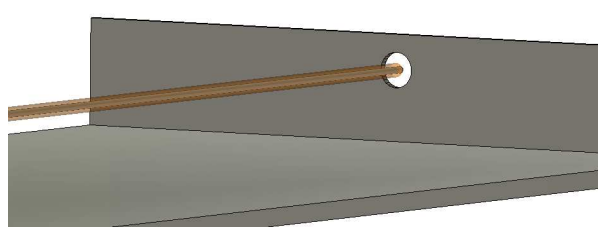

Fig. 3. A detail of the model of a single-wire line used in the numerical validation and in the derivation of the empirical error model (30).

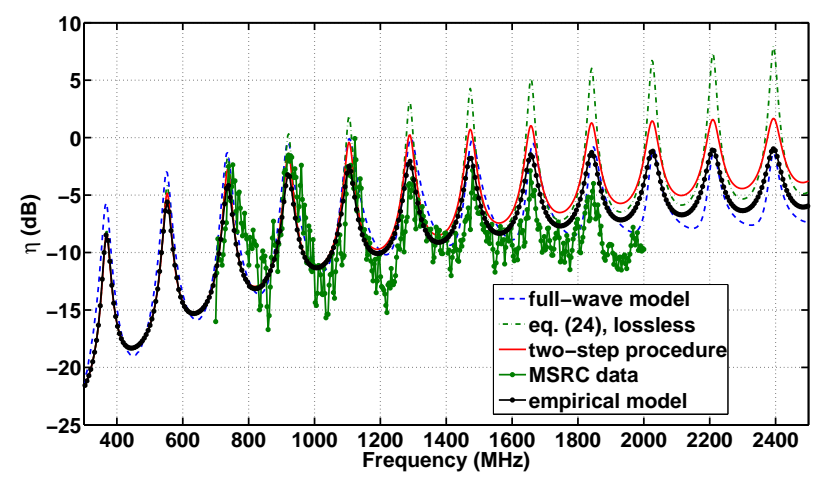

Fig. 4. Validation of (24) by comparison to full-wave numerical simulations and experimental data. The results prove the possibility to extend the model validity to higher-frequencies through the two-step procedure. The measurement configuration under which the experimental results have been obtained is described in Section V.

\section{NumeriCAL VALIDATION AND ACCURACY ANALYSIS}

It is now important to check the ability of the approach here described to predict the behaviour of actual lines: this is more of a test of fitness of TLT, rather than of the proposed model, since (24) holds as long as the currentdistribution (3) is valid. This task can be accomplished by means of full-wave numerical simulations. We focus on a single-wire structure, in order to identify under which conditions (24) fails, and thus to give a rule for the characterization of the approximation error it involves. To this end, a grand-total of 24 configurations was studied. All the numerical results have been obtained by using the Finite Integral Technique as implemented

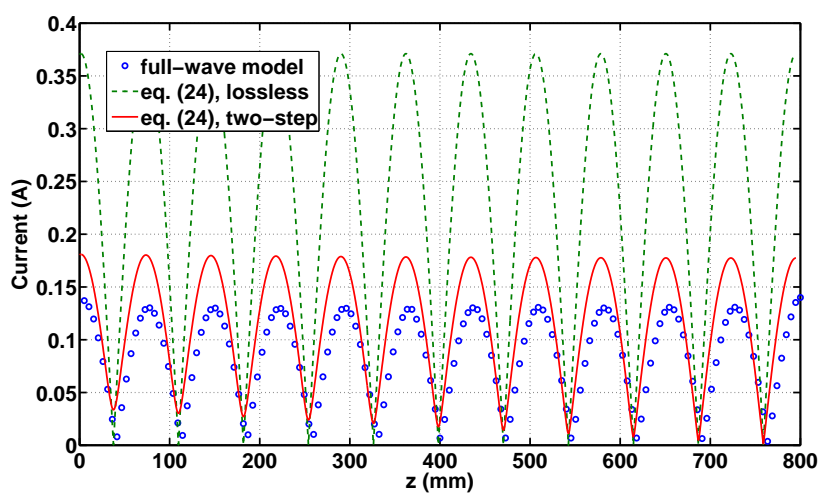

Fig. 5. The current distribution along the overhead wire for the example in Fig. 4, as computed at the TRP peak at $f=2.029 \mathrm{GHz}$. by CST's Microwave Studio. A single-wire line, as the one in Fig. 3, has been modelled for a wire height $h=\{20,30,50\} \mathrm{mm}$, a length $\mathscr{L}=80 \mathrm{~cm}$ and a load resistance $R_{L}=\left\{0,20,50,100,250,500,10^{3}, 10^{6}\right\} \Omega$. The numerically computed radiation efficiency $\eta$ has been obtained by assessing the power-loss in the structure through its scattering parameters, having paid attention to avoid any ohmic losses:

$$
\eta=1-\left|S_{11}\right|^{2}-\left|S_{21}\right|^{2}
$$

The radiation efficiency estimated by means of (24) will be hereafter referred to as $\hat{\eta}$. An example of these results is shown in Fig. 4, for $h=30 \mathrm{~mm}$ and $R_{L}=0 \Omega$ : the lossless version of (24) works rather well as long as $\hat{\eta}$ is well below the unity. This is directly linked to having required negligible radiation losses. Conversely, the two-step procedure allows a better estimation of $\eta$. The disagreements in the lower frequency-range are due to the fact that the line is electrically very close to the ground-plane. In this case the TRP is dominated by the vertical risers contribution, since they behave as electrically-short vertical monopoles.

It is also interesting to check the accuracy of the proposed model in the evaluation of the current-distribution. An example is shown in Fig. 5, for the configuration of Fig. 4 , at $f=2.029 \mathrm{GHz}$; this corresponds to one of the TRP peaks. These results show how the two-step procedure can account for the modification of the input impedance of the line, and thus of the current-distribution.

The numerical results were then compared to (24), relating the error $\hat{\eta}-\eta$ to $\hat{\eta}$. A typical example of error distribution is shown in Fig. 6a, for $h=50 \mathrm{~mm}$, $\mathscr{L}=80 \mathrm{~cm}$ and $R_{L}=0 \Omega$. Figure $6 \mathrm{~b}$ shows how the two-step procedure affects the error distribution: indeed, the procedure is effective in reducing the non-physical overestimation of $\hat{\eta}$.

Concerning the estimation error for the lossless case, the results in Fig. 6 are representative of the proposed model; the standard deviation for a given value of $\hat{\eta}$ is typically about $0.5 \mathrm{~dB}$ around the average error. For this reason, and for the sake of clarity, only the average error distributions are hereafter shown.

The distributions thus obtained are collected in Fig. 7; it is clear from these results that the overestimation of the TRP through TLT and equation (24) follows a law that is monotonous and well fitted by a quadratic curve. Interestingly, the fitting curve can fairly represent all of the load and geometry configurations considered; the dependence on the actual configuration is indeed indirectly accounted for by recalling that configurations with low radiation efficiency will produce error curves in the lower-end of Fig. 7, while highly resonant lines will sweep the entire span of values. In other words, currentdistributions associated to low radiations will be fairly representative of the reality, whereas in the case of high radiation levels the TRP associated will be not correct, since underestimating the importance of radiation losses.

The fitting curve shown in Fig. 7 is given by:

$$
\hat{\eta}-\eta=\left(e_{0}+e_{1} \hat{\eta}\right)^{2} \quad \hat{\eta}>-12.8,
$$




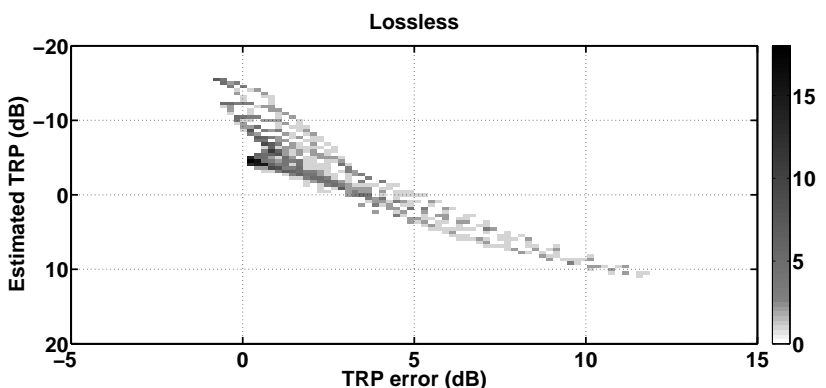

(a)
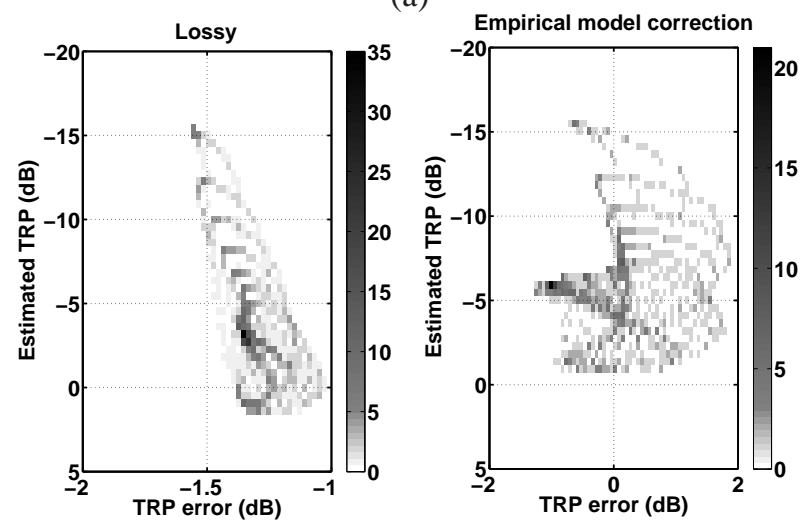

(b)

Fig. 6. Error distributions between the estimation of the TRP given by (24) and obtained through full-wave simulations (a) and after the application of the two-step procedure and of the empirical error model (b), accounting for radiation losses. The intensity of the dark tones indicates the frequency of occurrence for a given bin.

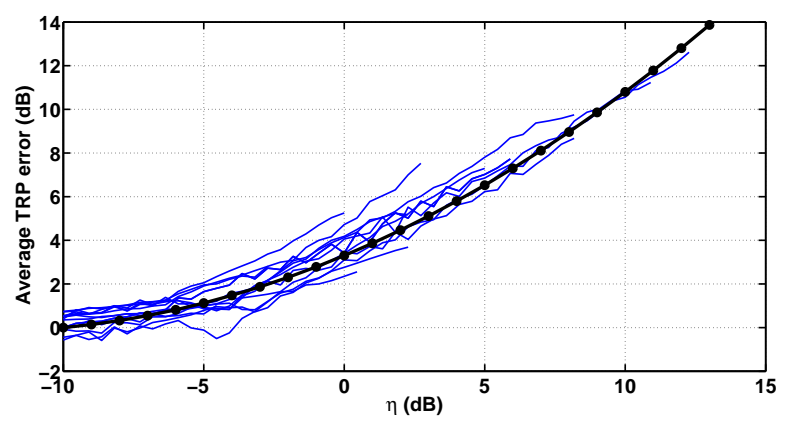

Fig. 7. Average error in the TRP given by (24). Several load resistances were considered, for the two cases $h=\{20,50\} \mathrm{mm}$. The parameters of the empirical model (solid line with dots) were obtained by fitting (30) to these data.

having considered $\eta$ and $\hat{\eta}$ as expressed in $\mathrm{dB}$; the model parameters are $e_{0}=1.82, e_{1}=0.15$. Although this result was apparently not derived from extensive simulations, the configurations studied covered all the possible cases; indeed, three parameters are responsible for the behaviour of the line, i.e., $k_{0} \mathscr{L}, k_{0} h$ and $\Gamma_{\mathrm{L}}$. The fact that the error was analyzed over a frequency-range spanning the entire range of validity of the TLT indicates that a few geometrical configurations hold enough information for all the possible cases (an example of the results obtained for a different structure is given in Fig. 8). By doing so for the entire spectrum of values of $\Gamma_{L}$ ensures that (30) is a general result. This will be shown to hold even in the case of MTLs in Section VIII.

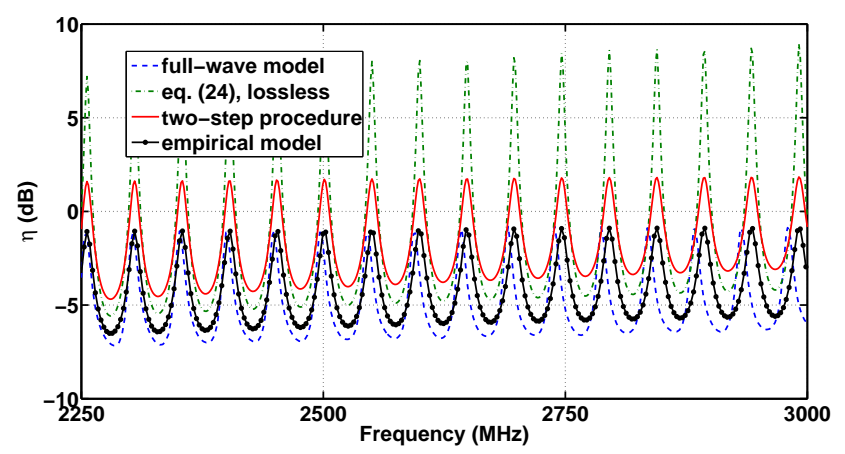

Fig. 8. Results obtained by applying the empirical model to a structure different from the original set of data: the example of a very long singlewire line $\left(h=3 \mathrm{~cm}, \mathscr{L}=3 \mathrm{~m}, \Gamma_{\mathrm{L}}=-1\right)$.

The fact that the error-law is monotonous can be exploited by using it to correct $\hat{\eta}$ in the lossless case. An example of the effects of such a correction is given in Figures 4 and 6b. Although not physically sound as the inclusion of radiation losses, (30) can be effectively used to extend the validity of (24), with no need to use the two-step procedure. This simple empirical law has no pretention of representing exactly the error between the lossless estimate and the actual TRP. As a matter of fact, the fitting has been derived over the averaged errors, and it is affected by a maximum fitting error of $1 \mathrm{~dB}$. Nevertheless, equation (30) accounts for the average error in the TRP estimation. Its merit is that of having been defined independently of the geometrical and electrical configuration. Besides allowing to avoid the two-step procedure in the single-wire case, this approach is fundamental for the MTL case described in Section VII, where the two-step procedure is not available. It is fundamental to understand that (30) does not operate over the current-distribution, but merely on the estimated TRP.

\section{EXPERIMENTAL VALIDATION IN AN MSRC}

As recalled in the Introduction, there are two ways of rapidly measuring the TRP of a line: through powerbalance methods or by using a MSRC. The problem with the first method is that it requires the knowledge of the entire scattering matrix of the line and that the TRP is not linearly related to the current distribution along the line. This means that a change in the termination loads cannot be accounted for by just recalculating a new scattering matrix from the measured one. The TRP estimated from power-balance methods is therefore valid just for the actual load configuration at the moment of the measurement. This means that the input impedances of the network analyzer need to be set to the actual values that the user wants to test as termination loads. Furthermore, superposition of effects is not an option here, due to the quadratic relationship between the scattering parameters and the TRP. This is an issue in the case of multiplesources configurations, in particular in the case of MTLs. Much simpler is the measurement of the TRP with an MSRC, using lumped loads.

A line like the one in Fig. 9 has been considered. The line was kept suspended by two vertical supports, 


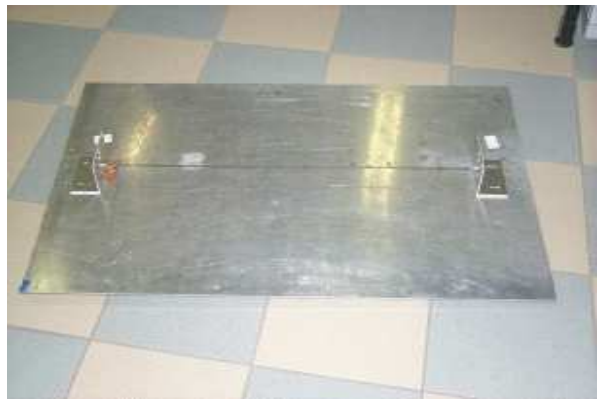

Fig. 9. The line used for the experimental validation.

whereas the line ends were connected to two SMA-type connectors. The first connector was used for loading the line with SMA-type loads, and the other one was connected to a coaxial cable for the line excitation.

The MSRC was used as described in [5]; the stirrer has a major role in ensuring the statistical independence of the data. It was set to perform a complete turn in $7 \mathrm{~s}$, by 360 finite steps for each frequency investigated. Since the MSRC dimensions were $1.9 \times 2.65 \times 2.9 \mathrm{~m}$, the minimum frequency for which the chamber can be regarded as overmoded is about $700 \mathrm{MHz}$. The line was fed through a function generator kept outside the MSRC and connected to the line through a coaxial cable passing through a via in the MSRC wall. In the same way a horn was used as the receiving antenna, connected to a spectrum analyzer outside the MSRC.

The resulting $P_{\text {line }}$ obtained with this procedure is not yet a correct evaluation of the TRP: in fact, it includes insertion losses. These include cable losses, mismatches, and the MSRC insertion losses (its Q being finite). Because of this, the line was substituted by another horn antenna (identical to the receiving one), and the entire procedure was repeated, thus obtaining a reference measurement $P_{\text {ref }}$.

These two measurements can be represented as follows, by decomposing them into several basic contributions:

$$
\begin{aligned}
P_{\text {line }} & =P_{\mathrm{av}}\left(1-\left|\Gamma_{\text {line }}\right|^{2}\right) T_{\text {line }} T_{\mathrm{IL}}, \\
P_{\text {ref }} & =P_{\mathrm{av}}\left(1-\left|\Gamma_{\text {horn }}\right|^{2}\right) T_{\text {horn }} T_{\mathrm{IL}},
\end{aligned}
$$

where $P_{\mathrm{av}}$ is the available power provided by the power generator and $\Gamma_{\text {line }}$ and $\Gamma_{\text {horn }}$ are the reflection coefficients as seen from the input port of, respectively, the line and the horn antenna. The term $T_{\text {line }}$ is a transfer function defined as the ratio between the power radiated by the line (i.e., its TRP) and the active power going into it through its input ports. In other words, $T_{\text {line }}$ accounts for the radiation efficiency of the line. In the same way the transfer function $T_{\text {horn }}$ has been defined for the horn antenna. Finally, $T_{\mathrm{IL}}$ is the insertion loss accounting for all the losses between the actual TRP and the power measured by the spectrum analyzer. The TRP can thus be expressed as $P_{r}=P_{\text {av }}\left(1-\left|\Gamma_{\text {line }}\right|^{2}\right) T_{\text {line }}$.

Because of the way it was defined, $T_{\text {horn }}$ can be assumed to be equal to one, thus obtaining the radiation efficiency $\eta$ of the line:

$$
\frac{P_{r}}{P_{\text {av }}}=\eta=\frac{P_{\text {line }}}{P_{\text {ref }}}\left(1-\left|\Gamma_{\text {horn }}\right|^{2}\right) .
$$

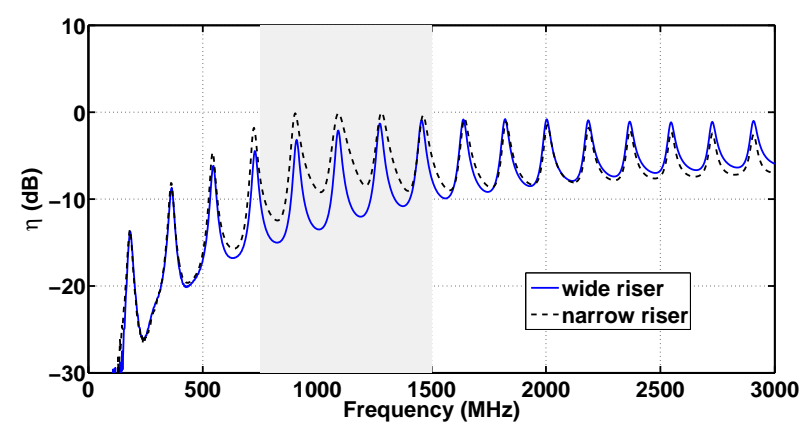

Fig. 10. Numerical results for the TRP of a $80 \mathrm{~cm}$ long single-wire line, $h=3 \mathrm{~cm}$, for two widths of the vertical risers, respectively $200 \mathrm{~mm}$ and $50 \mathrm{~mm}$. The shaded area corresponds to the frequency-range where vertical-risers are expected to radiate effectively.

Attention should be paid to the fact that $\Gamma_{\text {horn }}$ is meant to be measured for the actual setup configuration, i.e., with the horn antenna inside the MSRC, because reflections modify its input impedance.

Some experimental results are shown in Fig. 4: the line under test was short-circuited at its far-end, with $h=3 \mathrm{~cm}, \mathscr{L}=80 \mathrm{~cm}$; the diameter of the metallic core of the wire was $0.7 \mathrm{~mm}$, its PVC coating $0.15 \mathrm{~mm}$ thick and the ground-plane dimensions were $60 \mathrm{~cm}$ by $100 \mathrm{~cm}$. The TRP was studied in the frequency-range $700 \mathrm{MHz}$ to $2 \mathrm{GHz}$, hence considering the line as electrically long $(\mathscr{L} / \lambda \simeq 1.9$ at $700 \mathrm{MHz}$ ). These results highlight how radiation losses get more important as the frequency increases, in particular for reactive loads leading to a resonant behaviour. As expected, radiation losses can be neglected as long as $h / \lambda \ll 1$. In fact, below $1 \mathrm{GHz}$ the results obtained by directly applying (24) are very satisfying. In general, the experimental results shown in Fig. 4 are well reproduced by the proposed model, with an average absolute error attaining $2 \mathrm{~dB}$ around the TRP minima. Major discrepancies occurring between $750 \mathrm{MHz}$ and $1500 \mathrm{MHz}$ are due to the presence of the vertical risers. Indeed, the currents flowing through them radiate a field that is not negligible as soon as the length of the current paths correspond to a quarter of wavelength. In these cases, the current distribution effectively radiates, being in a monopole-like configuration. Hence, the vertical risers can be expected to radiate more strongly over the frequency-range $f \in c /\left(4 h_{i}\right)[0.5,1]$, since the current paths typically have a length in the range $[h, 2 h]$ (see Fig. 10).

Moreover, the use of narrow vertical risers imposes, in their proximity, a deformation of the currents path over the ground plane (current channeling). This means that the assumption of line uniformity is no more valid. This can be accounted for by looking for an equivalent model of the line ends, and to consider just the central portion of the line as a uniform structure, as suggested in [12].

It is interesting to note that the experimental data are characterized by a stronger attenuation than the numerical ones. This is likely due to two reasons: ohmic losses were neglected in the numerical simulations, and contact resistances were certainly not negligible between the vertical risers and the ground plane, these not being soldered. 


\section{AN APPLICATION TO MTLS}

Although (24) holds just for a single-wire line, it can be usefully employed for an MTL, too. Indeed, in EMC the most dramatic effects regarding the radiated emission/immunity problems are due to the commonmode $(\mathrm{CM})$ current, or bulk current $I_{b}(z)$. Let us consider the modal description for the current-distribution along an $N$-wire MTL [20]:

$$
\begin{aligned}
\mathbf{I}(z) & =\mathbf{T}\left[\mathbf{P}^{+}(z) \mathbf{I}_{m 0}^{+}-\mathbf{P}^{-}(z) \mathbf{I}_{m 0}^{-}\right], \\
I_{b}(z) & =\sum_{i=1}^{N} I_{i}(z)=\mathbf{Q} \mathbf{I}_{m}(z) \\
& =\sum_{i=1}^{N} \sum_{k=1}^{N} T_{i k} I_{m, k}(z),
\end{aligned}
$$

where $\mathbf{T}$ is a square matrix relating the modal currents to the physical ones, $\mathbf{P}^{ \pm}(z)=\operatorname{diag}(\exp (\mp \boldsymbol{\gamma} z))$ are the propagation matrices, $\gamma$ is the vector of the line propagation constants, and $\mathbf{I}_{m 0}^{ \pm}$are the excitation factors for the forward- and backward-travelling modal-currents. The row-vector $\mathbf{Q}$ relating the modal currents to the bulk one is defined as the sum of the row-vectors $\mathbf{T}_{i}$ constituting the matrix $\mathbf{T}$ :

$$
\mathbf{Q}=\sum_{i=1}^{N} \mathbf{T}_{i}
$$

The far-field behaviour of the EM field radiated by an MTL is mainly dominated by the CM current, as long as the line conductors are "packed" together. With reference to Fig. 1, in the context of this work a "packed" line requires $k_{0}\left|\bar{h}-h_{i}\right| \ll 1$ and $k_{0}\left|\bar{d}-d_{i}\right| \ll 1 \forall i \in[1, N]$, as well as $h_{i} \simeq \bar{h}$, where $\bar{h}$ and $\bar{d}$ stand for the mean values of, respectively, $h_{i}$ and $d_{i}$.

Let us consider a uniform MTL: the magnetic field can be expressed, in the far-field, as:

$$
\begin{aligned}
& \mathbf{H}(\rho, \vartheta, \varphi)=\hat{\boldsymbol{\varphi}} \frac{k_{0}}{2 \pi \rho} \sin \vartheta \sin \left(k_{0} h_{i} \hat{\boldsymbol{x}} \cdot \hat{\boldsymbol{\rho}}\right) \mathrm{e}^{-\gamma_{0} \rho} \\
& \sum_{i=1}^{N} \mathrm{e}^{-\gamma_{0} d_{i} \hat{\boldsymbol{y}} \cdot \hat{\boldsymbol{\rho}}} \int_{0}^{\mathscr{L}} \sum_{k=1}^{N} T_{i k} I_{m, k}(z) \mathrm{e}^{-\gamma_{0} z \hat{\boldsymbol{z}} \cdot \hat{\boldsymbol{\rho}}} \mathrm{d} z
\end{aligned}
$$

which can be approximated, for a packed line as:

$$
\begin{aligned}
\mathbf{H}(\rho, \vartheta, \varphi) \simeq & \hat{\boldsymbol{\varphi}} \frac{k_{0}}{2 \pi \rho} \sin \vartheta \sin \left(k_{0} \bar{h} \hat{\boldsymbol{x}} \cdot \hat{\boldsymbol{\rho}}\right) \mathrm{e}^{-\gamma_{0} \rho} . \\
& \cdot \mathrm{e}^{-\gamma_{0} \bar{d} \hat{\boldsymbol{y}} \cdot \hat{\boldsymbol{\rho}}} \int_{0}^{\mathscr{L}} I_{b}(z) \mathrm{e}^{-\gamma_{0} z \hat{\boldsymbol{z}} \cdot \hat{\boldsymbol{\rho}}} \mathrm{d} z .
\end{aligned}
$$

This result derives from (35), and shows that the main contribution to the radiated field is due to the CM current. The importance of this result lays in the fact that whenever the current-distribution of the CM can be approximated by (3), the results obtained for the single-wire configuration can be applied to a multi-wire one. For these cases the single-wire current-distribution of the CM can be derived from the modal description as:

$$
\begin{aligned}
I_{b}(z) & =I_{b 0}^{+} \mathrm{e}^{-\gamma_{b} z}-I_{b 0}^{-} \mathrm{e}^{+\gamma_{b} z}, \\
I_{b 0}^{ \pm} & =I_{m 0, j}^{ \pm} \sum_{i=1}^{N} T_{i j},
\end{aligned}
$$

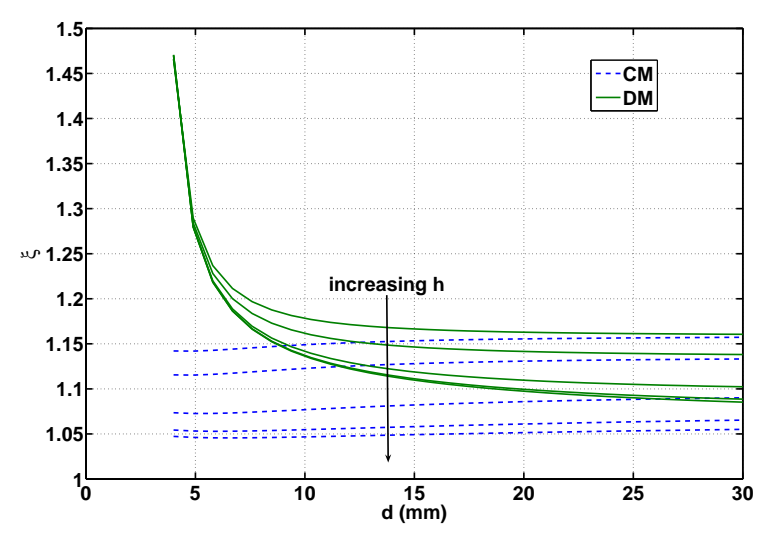

Fig. 11. Common-mode and differential-mode normalized propagation constants $\xi$ for a two-wire line as a function of wire distance $d$. The wires were at a varying distance $d, h_{1}=h_{2}=\{3.5,5,10,20,30\} \mathrm{mm}$, conductors radii $r_{1}=r_{2}=1 \mathrm{~mm}$, dielectric coating $1 \mathrm{~mm}$ thick with $\epsilon_{r}=3.5$.

where $\gamma_{b}$ corresponds to the CM propagation constant in $\gamma$, as its j-th element. Since the medium surrounding the metallic wires is generally not homogeneous, the vector of the propagation constants is expected to have elements all different from each other. Due to the fact that the transversal topography of the electromagnetic field associated to the CM has force-lines mostly in air, its propagation constant is very near to that of air. Conversely, differential modes present the opposite situation, so that the $\mathrm{CM}$ is easily recognizable.

On the other hand, for a homogeneous medium $\gamma_{i}=$ $\gamma \forall i \in[1, N]$, so that the previous procedure is no longer available. Thus, $\mathbf{P}^{ \pm}(z)=\exp (\mp \gamma z) \mathbb{I}$ so that:

$$
I_{b}(z)=\mathbf{Q}\left[\mathbf{I}_{m 0}^{+} \mathrm{e}^{-\gamma z}-\mathbf{I}_{m 0}^{-} \mathrm{e}^{+\gamma z}\right]=I_{b 0}^{+} \mathrm{e}^{-\gamma z}-I_{b 0}^{-} \mathrm{e}^{+\gamma z} .
$$

In this case the single-wire representation of the currentdistribution of the CM is exact, and no approximation is needed. Moreover, the above relationship can be also invoked for MTLs with dielectrically coated wires, as long as they are sufficiently wide apart to behave as a line in air. An example supporting this approach is shown in Fig. 11, where the normalized propagation constants $\xi_{i}$ have been computed for a two-wire line, as a function of the wires distance $d$ and their height $h$. These results show that the condition $\xi \simeq 1$ is not just met by the CM, but also the DMs converge quite rapidly towards this value.

\section{EXTENSION TO A MULTICONDUCTOR LINE}

As conductors get wider apart, the contributions from modes other than the CM can be no longer neglected.

In order to overcome this limitation, the same approach previously applied to the single-wire line can be utilized for a multiwire structure. In this case the overall powerdensity flux to be integrated is no longer generated by a single conductor, but rather by a set of $N$ parallel conductors, for which the overall far-field magnetic field $H_{t}$ is given by the contributions of the $N$ conductors:

$$
H_{t}=\sum_{i=1}^{N} H_{i}
$$


where $H_{i}$ is the contribution to the magnetic field due to the current-distribution along the $i$-th conductor. Hereafter, the arguments $\rho, \vartheta$ and $\varphi$ will be dropped for the sake of clarity. Now, (2) can be recast as follows

$$
P_{r}=\frac{1}{2} \int_{\Omega} \zeta_{0}\left|H_{t}\right|^{2} \mathrm{~d} \Omega=\frac{1}{2} \int_{\Omega} \zeta_{0}\left|\sum_{i=1}^{N} H_{i}\right|^{2} \mathrm{~d} \Omega .
$$

The summation can be brought out of the modulus symbol by means of the following equivalence:

$$
\left|\sum_{i=1}^{N} H_{i}\right|^{2}=\sum_{k=1}^{N}\left|H_{k}\right|^{2}+2 \sum_{t>k} \operatorname{Re}\left\{H_{k} H_{t}^{*}\right\} .
$$

This expression allows the integral in (43) to be broken down into simpler integrals; by defining the quantity $P_{k t}$ as

$$
P_{k t} \triangleq \zeta_{0} \int_{\Omega} \operatorname{Re}\left\{H_{k} H_{t}^{*}\right\} \mathrm{d} \Omega,
$$

(43) can be rewritten as

$$
P_{r}=\frac{1}{2} \sum_{k=1}^{N} P_{k k}+\sum_{t>k} P_{k t}
$$

This more general approach incorporates the same result obtained in (24) for the single-wire configuration. In particular, in that case only the term $P_{11}$ is to be considered; this gives us the key for a physical interpretation of the terms in (46): the terms $P_{k k}$ yield the power that would be radiated by the current-distribution along the $k$-th conductor, were it in a single-wire configuration. For this reason, one can refer to these terms as singlewire contribution terms. In other words, the first sum in (46) neglects any interference between the EM fields generated by the different current-distributions along the $N$ conductors. Conversely, the terms $P_{k t}$ just take into account the mutual interference between each pair of contributions. Therefore, these terms can be referred to as mutual terms.

Thanks to (46), the TRP for an MTL can be easily estimated once a solution for the generic term $P_{k t}$ in (45) is available. As for the single-wire case, the MTL is analyzed through a quasi-TEM approach, so that transmission-line theory can be applied. By employing the same modal description introduced in (35) and applied in (37), the following general result can be derived:

$$
\begin{aligned}
H_{k}= & \frac{k_{0} \sin \vartheta}{2 \pi \rho} \sin \left(k_{0} h_{k} \hat{x} \cdot \hat{\rho}\right) \mathrm{e}^{-\gamma_{0} d_{k} \hat{y} \cdot \hat{\rho}} . \\
& \cdot \sum_{i=1}^{N} T_{k i}\left(I_{m 0, i}^{+} \Delta_{i}^{+}-I_{m 0, i}^{-} \Delta_{i}^{-}\right),
\end{aligned}
$$

having defined

$$
\Delta_{i}^{ \pm} \triangleq \frac{1-\mathrm{e}^{-\mathscr{L}\left(\gamma_{0} \cos \vartheta \pm \gamma_{i}\right)}}{\gamma_{0} \cos \vartheta \pm \gamma_{i}} .
$$

Expression (47) has been obtained by analytically integrating the current-distribution over the entire length $\mathscr{L}$ of the line. Now, this expression is too complex to fit the target of this work, i.e., to propose a simple analytical solution. Therefore a simplifying hypothesis is introduced, by assuming $\gamma_{i} \simeq \gamma_{0}$. It was shown in the previous
Section that this hypothesis can be met even with coated wires, whenever they are sufficiently far away from each other. Therefore this assumption is not a limitation but it rather identifies an open line, i.e., a scenario that is thus complementary to the packed-line case.

By applying the above hypothesis we get $\Delta_{i}^{ \pm} \simeq \Delta^{ \pm}$, by substituting $\gamma_{i}$ with $\gamma_{0}$. We are now in a position to effectively simplify (47):

$$
\begin{aligned}
H_{k}= & \frac{k_{0} \sin \vartheta}{2 \pi \rho} \sin \left(k_{0} h_{k} \hat{x} \cdot \hat{\rho}\right) \mathrm{e}^{-\gamma_{0} d_{k} \hat{y} \cdot \hat{\rho}} . \\
\cdot & {\left[\Delta^{+} I_{k}^{+}(0)-\Delta^{-} I_{k}^{-}(0)\right] }
\end{aligned}
$$

where

$$
I_{k}^{ \pm}(0)=\sum_{i=1}^{N} T_{k i} I_{m 0, i}^{ \pm}
$$

are the forward- and backward-travelling wave contributions to the current-distribution along the $k$-th wire, as computed at its left-end.

By inserting (49) into (45) and basically following the same procedure applied for the single-wire configuration, a general solution for the term $P_{k t}$ can be derived, obtaining:

$$
\begin{aligned}
P_{k t} & =\frac{\zeta_{0}}{4 \pi^{2}} \sum_{n=1}^{\infty} a_{n}\left[\left(\tau^{\prime} \kappa_{c}-\sigma^{\prime}\right) S_{2 n+1}+\right. \\
& +2 \sigma^{\prime} S_{2 n-1}+ \\
& -2 \sigma^{\prime} \kappa_{c} \psi_{n}+ \\
& \left.+\left(\sigma^{\prime} \kappa_{c}-\tau^{\prime}-\sigma^{\prime} \kappa_{s} k_{0} \mathscr{L} / n\right) \psi_{n+1}\right],
\end{aligned}
$$

where $\tau^{\prime}=\operatorname{Re}\left\{\gamma \exp \left(-\gamma_{0} \mathscr{L}\right)+\delta \exp \left(+\gamma_{0} \mathscr{L}\right)\right\}, \sigma^{\prime}=$ $\operatorname{Re}\{\alpha+\beta\}$ and

$$
\begin{aligned}
\alpha & =I_{m 0, k}^{+} I_{m 0, t}^{+*} \\
\beta & =I_{m 0, k}^{-} I_{m 0, t}^{-*} \\
\gamma & =I_{m 0, k}^{+} I_{m 0, t}^{-*} \\
\delta & =I_{m 0, k}^{-} I_{m 0, t}^{+*}
\end{aligned}
$$

The terms $\psi_{n}(\cdot)$ and the coefficients $S_{n}$ have been already introduced for the solution to the single-wire case in (25) and (26). The series expansion coefficients $a_{n}$ depend on the indexes $k$ and $t$ and are defined as

$$
a_{n}=-a_{n-1}\left(\frac{k_{0}}{2 n}\right)^{2} \frac{\rho_{-}^{2 n}-\rho_{+}^{2 n}}{\rho_{-}^{2(n-1)}-\rho_{+}^{2(n-1)}},
$$

where $\rho_{ \pm}^{2}=\left(d_{k}-d_{t}\right)^{2}+\left(h_{k} \pm h_{t}\right)^{2}$ and $c_{1}=2 \pi k_{0}^{2} h_{k} h_{t}$.

The solution proposed in (46) is interesting also because of the possibility to apply a topological analysis to the problem of radiation from an MTL. Indeed, the $P_{k t}$ terms allow to identify the dominant contributions to the TRP, hence the subgroups of wires (and their electrical configuration due to the end loads) that are more critical to radiated immunity/emissions. This possibility should provide a better insight into the mechanisms of radiation and its minimization for the case of a uniform transmission line, in particular during the design phase.

Unlike the single-wire solution (24), no two-step procedure can be envisaged for the moment. Indeed, since radiation losses have a very different physical origin 


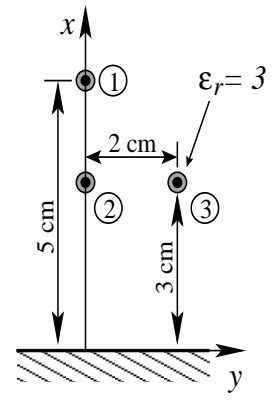

(a)

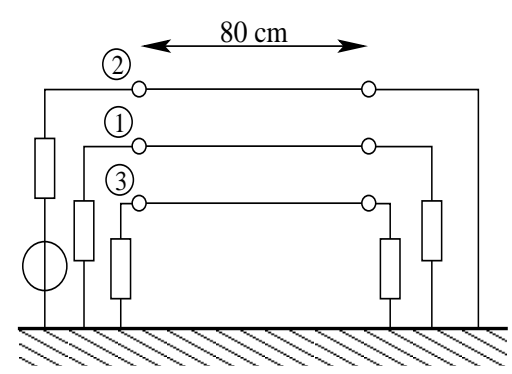

(b)
Fig. 12. The cross-section of the MTL (a) and the electrical configuration used during the experimental validation (b). The active wire is identified by the tag "2". All the loads are $50 \Omega$ resistances.

than ohmic ones, an eventual radiation resistance matrix would depend not just on the geometry of the line (like for ohmic losses), but also on the current distribution. Although a clear relationship can be defined for a singlewire line, linking the TRP and the current distribution through the radiation resistance (27), the extension of this approach to a multiconductor configuration is not trivial. Researches are currently in progress, with the aim to propose an empirical redistribution of the radiation losses for an MTL configuration. Nevertheless, the use of the empirical error model (30) provides a zero-order correction of the TRP estimation, as already shown for the single-wire configuration and proved in further tests on MTL structures (see next Section).

The assumption $\xi_{i} \simeq 1$ does not exclude the possibility to have a finite conductivity for the ground-plane, as long as the wave propagation can be regarded as a perturbation of the ideal case. This hypothesis could reduce the validity of (51) in the high-frequency range for this specific case.

\section{VALIDATION FOR AN OPEN MTL}

In order to check the validity of the results provided by (46) and (51), the same procedure used for the validation of the single-wire configuration will be followed. Rather than exciting several conductors at the same time by means of a multiwire excitation, only one conductor is connected to the power generator, while the others will be excited by coupling (see Fig. 12). This choice is due to the practical difficulties that arise when trying to provide a multiwire excitation external to the MSRC. Therefore just one conductor is active, whereas the others act as passive ones, modifying the radiation properties of the entire system.

The experimental estimation of the TRP was carried out as already described in Section V. The results obtained, shown in Fig. 13, prove the ability of (51), when applied with the empirical model (30), to correctly predict the total power radiated by a uniform MTL. As for the singlewire model, there is a disagreement over the frequencyrange where the vertical-risers act as tuned monopole antennas. Conversely, the agreement with the rest of the experimental data is fairly good, but for a stronger attenuation that was already pointed out during the validation

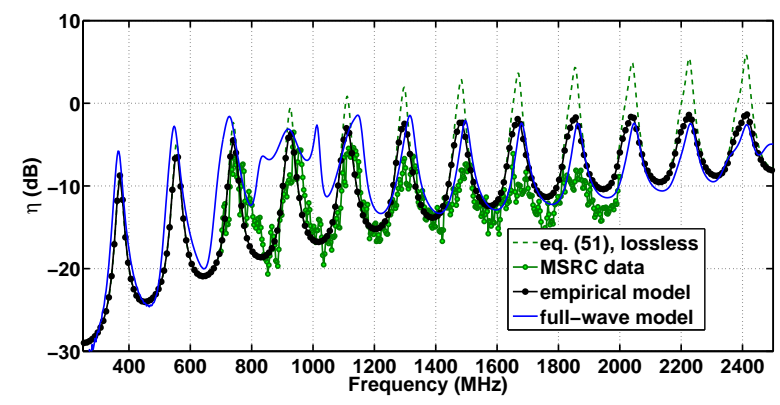

(a)

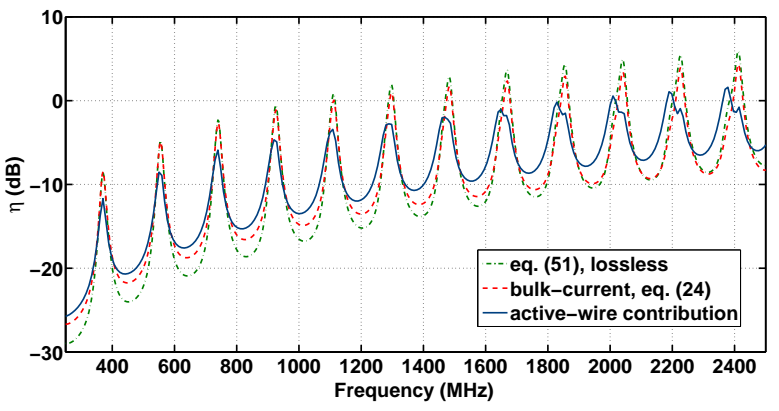

(b)

Fig. 13. Validation of (51) for the line in Fig. 12 by comparison to fullwave numerical simulations and experimental data. The results prove the possibility of using (24) even for a non-packed line. The empirical error model is also shown to give very good results, with no need to assess radiation losses.

of the single-wire model. The TRP obtained for the bulkcurrent is very close to the results yielded by all the propagating modes, although major differences appear near the minima of the TRP, because the assumption of packed-line are not fully satisfied.

Figure 13 also shows the TRP that would be generated by the current-distribution along the active conductor, should it be in a single-wire configuration. The comparison highlights that the passive conductors play a nonnegligible role in the overall TRP. This implies that the actual configuration used for the experimental validation is not a degenerate case of MTL.

A remarkable result of this validation is that the use of the empirical error model can be extended to MTL. This conclusion is supported by the results obtained through many other tests, some of which are shown in Fig. 14. It is interesting to notice how the empirical model can always reduce the TRP error to values below $2 \mathrm{~dB}$. Moreover, these tests also show how the bulk-current model may fail in open configurations. In particular, the only fact of supplying the line of Fig. 12 on the highest wire (tag no. 1) leads to underestimating the TRP of $5 \mathrm{~dB}$ in the lower frequency-range (Fig. 14a). Similar results are shown in Fig. 14e, where the two lower conductors, although conveying very low current levels with respect to the higher one, affect the power radiated by the active wire in a non-negligible way. These results demonstrate the usefulness of (51): a single-wire model cannot account for the actual excitation of a wire-bundle when it is not packed. 


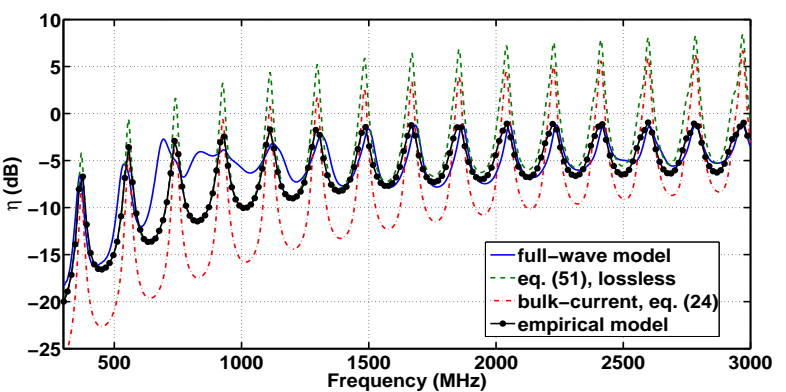

(a)

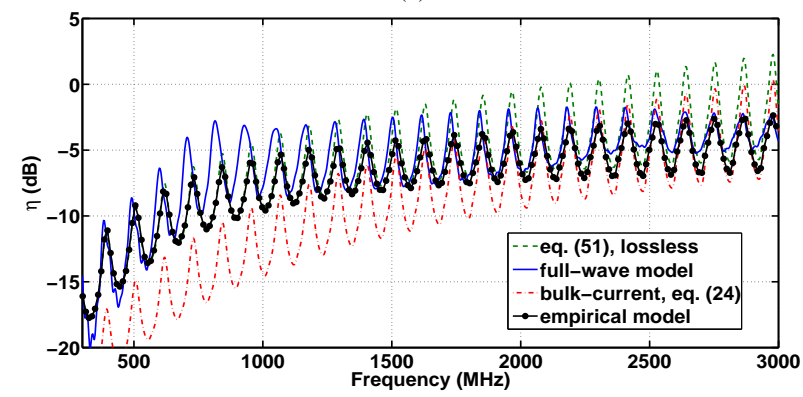

(b)

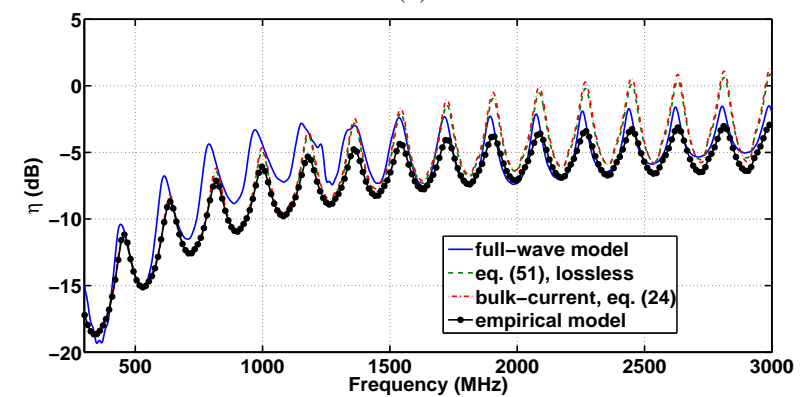

(c)

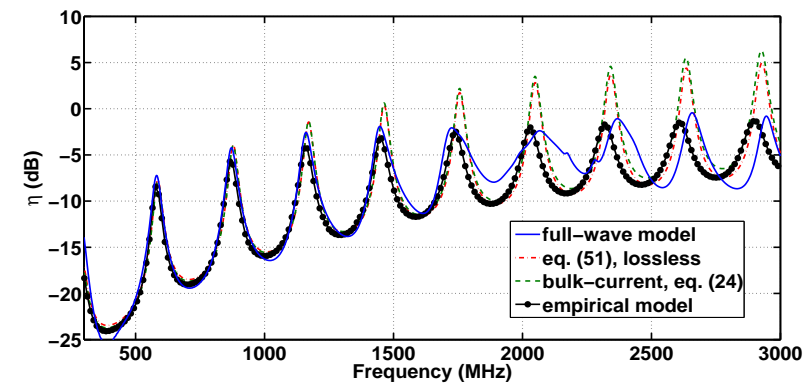

(d)

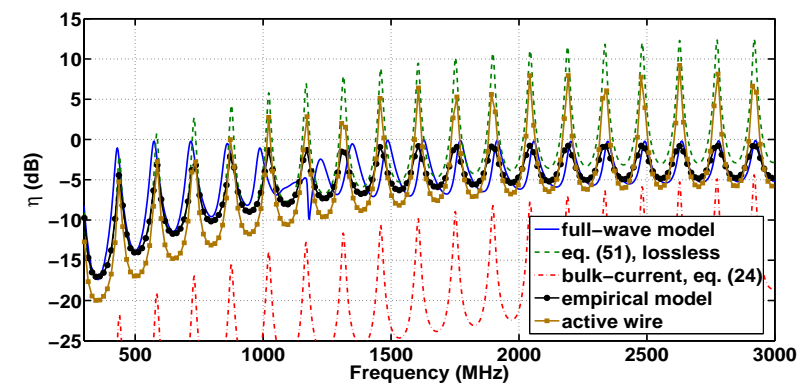

(e)

Fig. 14. Numerical validation results for an MTL: a) same configuration than in the previous validation case, but with wire no. 1 now active (see Fig. 12), and b) with $\mathscr{L}=1.3 \mathrm{~m}$ and a $1 \mathrm{k} \Omega$ load resistance at the far-end of the active wire; c) three-wire configuration with $d=$ $\{-25,-5,25\} \mathrm{mm}, h_{i}=50 \mathrm{~mm}, \mathscr{L}=80 \mathrm{~cm}$, active wire at the furthest left, all loads set as $50 \Omega$ resistances but for the one at the far-end of the active wire, set at $1 \mathrm{k} \Omega$, d) same structure, but with $h_{i}=20 \mathrm{~mm}, \mathscr{L}=50 \mathrm{~cm}$, active wire short-circuited at its far-end; e) same configuration as in c) but with $d=\{-20,0,20\} \mathrm{mm}, h_{i}=$ $\{50,5,5\} \mathrm{mm}, \mathscr{L}=1 \mathrm{~m}$, all loads set as $50 \Omega$ resistances but for the one at the far-end of the active wire, set at $0 \Omega$. All configurations have wire diameters of $0.7 \mathrm{~mm}$, with PVC coating $0.15 \mathrm{~mm}$ thick.

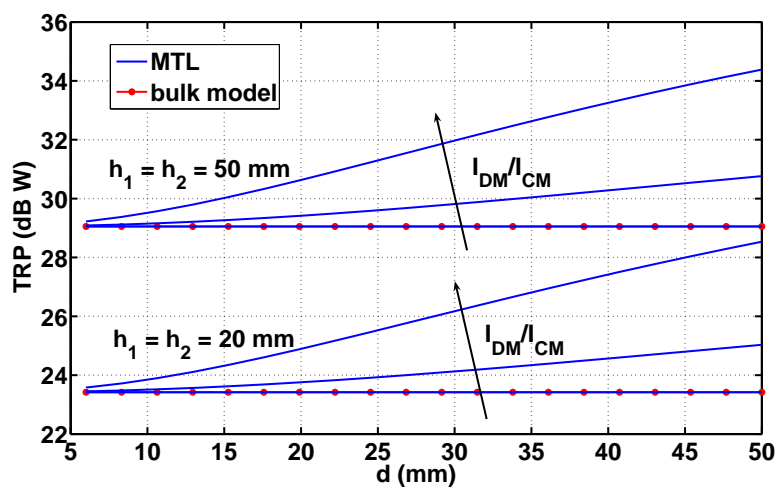

Fig. 15. TRP computed from (24) and (51) for a two-wire line at $f=2 \mathrm{GHz}$, as a function of wire distance $d$; the wires were not coated, with $r_{1}=r_{2}=1 \mathrm{~mm}, h_{1}=h_{2}=\{20,50\} \mathrm{mm}$ and $\mathscr{L}=1 \mathrm{~m}$. Modal currents corresponding to the CM and the DM were set to the ratios $I_{\mathrm{DM}} / I_{\mathrm{CM}}=\{1,2,4\}$.

\section{CONSIDERATIONS ON THE USE OF EQUATIONS (24) AND (51)}

The previous Sections have highlighted how (24) and (51) are not just related to two different electrical configurations, i.e., a single-wire and a multiconductor line, but rather to two different operating modes. Indeed, for a given MTL, the TRP can be estimated with one of these two equations, depending on whether radiation mechanisms are dominated by the CM or by the DMs propagating along the line, and not just depending on the number of conductors. A quantitative example of this interplay is given in Fig. 15, where a two-wire line with $h_{1}=h_{2}$ is considered. The distance between the two wires has been varied in order to assess the accuracy of the bulk-current model when the line conductors are not packed. Since the performances of (24) and (51) depend on the relative importance of the $\mathrm{CM}$ or the DM, the current distribution along the line was imposed by directly setting the modal current coefficients $I_{m 0}^{ \pm}$, so to have a certain ratio between the CM and DM currents. The wires were considered not coated, in order to avoid any approximation introduced by the hypothesis at the base of the two analytical solutions; the results given by (51) are thus exact and can be considered as a reference. The results in Fig. 15 show that even for distances that are electrically non-negligible, the bulk approach provides accurate results even for a ratio $I_{\mathrm{DM}} / I_{\mathrm{CM}}=2$. This is due to the well-known inefficient radiation mechanisms involved with DMs. Conversely, for $I_{\mathrm{DM}} / I_{\mathrm{CM}}=4$ the bulk-current model approach is valid as long as $d / \lambda \ll 1$ ( $d<15 \mathrm{~mm}$ at $2 \mathrm{GHz}$ ). Otherwise (51) could not be avoided even for a packed line.

\section{SUMMARY}

We have presented two analytical solutions for the TRP for an MTL above a ground-plane. The solutions, expressed as series expansions, appear to be fast-convergent, already yielding a good estimate with just one term. The novelty of the proposed models lies in the fact that they do not require the line to be electrically short, nor electrically close to the ground-plane. Besides, being based upon TLT, 
they are a natural extension to it; for instance, simulators based on TLT already have access to modal data regarding the propagation along a transmission-line, so that the actual implementation of these models has no additional costs.

The first part of this paper has dealt with the singlewire configuration, showing that it is possible to cover a very wide frequency-range by means of a two-step procedure. To this end, we defined an estimator for the radiation losses, which was subsequently used for assessing the attenuation introduced in the propagation along the line. The validity of this approach was checked through numerical and experimental investigations; the by-product of this validation was the definition of an empirical model for evaluating the overestimation of the TRP as computed with (24). This model proved to be configuration-independent, thus extremely simple to be applied.

In the second part of this work we introduced an extension of the model to open MTLs, where the bulkcurrent model may fail. In this case too, the experimental results have confirmed the validity of the proposed model, so that it can be usefully employed for the analysis of open lines. The empirical error model was shown to be still valid in the MTL case; it thus extends the range of validity of (51) to MTLs with high radiation efficiencies, with typical errors of about $1 \mathrm{~dB}$ and a maximum one of $3 \mathrm{~dB}$.

\section{ACKNOWLEDGEMENTS}

The authors are grateful to Dr. Lamine Koné (IEMN/TELICE, Lille, France) for his invaluable support during the experimental validations. The authors also wish to acknowledge the important contribution given by the reviewers to the final quality of the paper.

\section{REFERENCES}

[1] P. Wilson, G. Koepke, J. Ladbury, C.L. Holloway, "Emission and immunity standards: replacing field-at-a-distance measurementes with total-radiated-power measurements", 2001 IEEE International Symposium on EMC, Volume 2 , 13-17 Aug. 2001

[2] D.A. Hill, G. Camell, K.H. Cavcey, G.H. Koepke, "Radiated emissions and immunity of microstrip transmission lines: theory and reverberation chamber measurements", IEEE Trans. on EMC, vol. 38, no. 2, May 1996

[3] J. Krogerus, K. Kiesi, V. Santomaa, "Evaluation of three methods for measuring total radiated power of handset antennas", IEEE Instrumentation and Measurements Technology Conference, Budapest, Hungary, May 21-23, 2001

[4] P.F. Wilson, D.A. Hill, C.L. Holloway, "On determining the maximum emissions from electrically large sources", IEEE Trans. on $E M C$, vol. 40, no. 1, February 2002

[5] K. Rosengren, P.-S. Kildal, C. Carlsson, J. Carlsson,"Characterization of antennas for mobile and wireless terminals in reverberation chambers: Improved accuracy by platform stirring", Microwave Opt. Technol. Lett., vol. 30, no. 6, September 2001

[6] J. Kraus, Antennas, McGraw-Hill Companies, 1988

[7] F.M. Tesche, M.V. Ianoz, T. Karlsson, EMC Analysis Methods and Computational Models, 1997, John Wiley \& Sons

[8] J.E. Storer, R. King, "Radiation resistance of a two-wire line", Proceedings of IRE, vol. 39, 1951

[9] J. Nitsch,S. Tkachenko, "Telegrapher equations for arbitrary frequencies and modes: Radiation of an infinite, lossless transmission line", Radio Science, vol. 39, 2004

[10] J.B. Nitsch, S.V. Tkachenko, "Complex-valued transmission-line parameters and their relation to the radiation resistance", IEEE Trans. on EMC, vol. 46, no. 3, August 2004
[11] A. Maffucci, G. Miano, F. Villone, "Full-wave transmission-line theory", IEEE Trans. on Magnetics, vol. 39, no. 3, May 2003

[12] S. Tkatchenko, F. Rachidi, M. Ianoz,"Electromagnetic field coupling to a line of finite length: theory and fast iterative solutions in frequency and time domains", IEEE Trans. on EMC, vol. 37, no. 4, November 1995

[13] R.G. Olsen, E.F. Kuester, D.C. Chang, "Modal theory of long horizontal wire structures above the earth, 2, properties of discrete modes", Radio Science, Vol. 13, No. 4, July-August 1978

[14] M. D'Amore, M.S. Sarto, "Simulation Models of a Dissipative Trasmission Line Above a Lossy Ground for a Wide Frequency Range - Part II: Multiconductor Configuration", IEEE Transactions on Electromagnetic Compatibility, Vol. 38, No.2, May 1996

[15] J.R. Wait, K.P. Spies, "On the Image Representation of the Quasi-Static Fields of a Line Current Source Above the Ground", Canadian Journal of Physics, Vol. 47, 1969

[16] J.R. Wait, Electromagnetic Wave Theory, Harper \& Row Publishers, New York, 1985

[17] Gradshteyn, Ryzhik, Table of Integrals, Series, and Products, Fifth edition, January 1994, Academic Press

[18] V. Daniele, M. Gilli, S. Pignari, "Spectral theory of a semi-infinite transmission line over a ground plane", IEEE Trans. on EMC, vol. 38, No. 3, August 1996

[19] D.O. Wendt, J.L ter Haseborg, " Radiation losses representation in the transmission-line theory", International Symposium on EMC, Rome, Italy, September 13-16, 1994

[20] C.R. Paul, Analysis of Multiconductor Transmission Lines, John Wiley \& Sons, 1994 